\title{
A novel therapy for granulomatous lobular mastitis: Local heat therapy
}

\author{
XINXIN CHEN ${ }^{1,2}$, WANGJIAN ZHANG ${ }^{3}$, QIUER YUAN ${ }^{1}$, XIAOWU HU ${ }^{1}$, \\ TING XIA ${ }^{1}$, TENGFEI CAO ${ }^{1}$, HAIXIA JIA ${ }^{1}$ and LEHONG ZHANG ${ }^{1}$ \\ ${ }^{1}$ Department of Breast Surgery, ${ }^{2}$ Guangdong Provincial Education Department, \\ Key Laboratory of Nano-Immunoregulation Tumor Microenvironment, The Second Affiliated Hospital, \\ Guangzhou Medical University, Guangzhou, Guangdong 510260, P.R. China; ${ }^{3}$ Department of Environmental Health Sciences, \\ University at Albany, State University of New York, Rensselaer, NY 12144, USA
}

Received January 3, 2020; Accepted June 17, 2020

DOI: $10.3892 /$ etm.2021.10590

\begin{abstract}
Granulomatous lobular mastitis (GLM) is a chronic inflammatory breast condition that is characterized by granulomatous inflammation. GLM remains a refractory disease due to its failure to respond to routine anti-inflammatory therapies and its high recurrence rate. Thus, the present study aimed to investigate the application of local heat therapy in GLM as a potential therapeutic strategy. The results revealed that the application of local heat therapy was associated with a shortened remission time for GLM, while the remission and recurrence rates were similar to those of existing therapies. The median first remission time following local heat therapy was significantly decreased compared with that following corticosteroid therapy (5.30 months vs. 11.27 months; $\mathrm{P}<0.05$ ). The remission rates were not significantly different between the local heat therapy (76.9\%), extensive excision $(90.4 \%)$ and the corticosteroid therapy $(85.7 \%)$ groups $(\mathrm{P}>0.05)$. In addition, the recurrence rates were not statistically different between the groups (local heat therapy, $8.3 \%$; extensive excision, $10 \%$; and corticosteroid therapy, $10 \%$; P $>0.05)$. The local heat therapy showed mild adverse effects and shortened healing times compared to the other therapies; however, further confirmation is required.
\end{abstract}

\section{Introduction}

Granulomatous lobular mastitis (GLM) is a rare, chronic inflammatory breast disease, which is primarily characterized by nonspecific lobulitis involving multiple lobules $(1,2)$.

Correspondence to: Professor Lehong Zhang, Department of Breast Surgery, The Second Affiliated Hospital, Guangzhou Medical University, 250 Changgang Road, Haizhu, Guangzhou, Guangdong 510260, P.R. China

E-mail: zhanglehong123@126.com

Key words: corticosteroid therapy, extensive excision, local heat therapy, granulomatous lobular mastitis, Corynebacterium kroppenstedtii
GLM is more common in postpartum females compared with nullipara females and the clinical manifestations and imaging findings at the early stage of the disease are similar to those of breast cancer $(3,4)$, consisting of a lump, pain in the breast, red and swollen skin, ulceration and even abscesses in severe cases (5). Due to the extended duration of the disease, recurrent ulceration and the formation of multiple sinuses, GLM results in poor quality of life of affected patients, and in certain cases, it even causes depression (6). thus, there is an urgent requirement to discover a safe, well-tolerated and inexpensive therapeutic option for GLM.

Previous studies have suggested that expanded excision $(7,8)$, corticosteroid therapy (8-11) and anti-tuberculosis therapy (12) may be effective treatment options for GLM; however, to the best of our knowledge, no consensus has been reached regarding the most effective treatment regimen. The aforementioned therapies have numerous side effects, such as Cushing's syndrome and immunosuppression following corticosteroid therapy, liver damage or peripheral neuritis caused by anti-tuberculosis drugs or deformities of the breast following expanded excision $(13,14)$. Therefore, discovering a novel treatment that has fewer and less traumatic side effects remains a major challenge in the treatment of GLM.

Corynebacterium kroppenstedtii has been considered the major pathogenic factor for GLM and also the major causative factor contributing to the unresponsiveness of patients to routine antibiotics and the recurrence (15-17). The present study investigated the effect of local heat therapy as a treatment for GLM. Local heat therapy has been used as an anti-inflammatory strategy for decades, with efficacy in several conditions (18); however, to the best of our knowledge, the present study was the first to report on the application of local heat therapy for GLM. The present study investigated the cure rate, recurrence rate and adverse effects of local heat therapy.

\section{Materials and methods}

Study population. Female patients newly diagnosed with GLM by core needle biopsy and treated by the Department of Breast Surgery, The Second Affiliated Hospital of Guangzhou Medical University (Guangzhou, China) between 
January 2015 and December 2018 were included in the study for retrospective analysis. This study was approved by the Ethics Review Board of Guangzhou Medical University and the Ethics Review Board of The Second Affiliated Hospital of Guangzhou. Written informed consents were provided by the study participants and/or their legal guardians (approval no. 2017-ky-ks-11). The exclusion criteria were as follows: i) Other types of non-lactating mastitis; ii) failure of biopsy or pathological diagnosis; iii) incomplete medical records; and iv) a follow-up time of $<3$ months.

Patients were divided into three groups based on their treatment protocol: i) Corticosteroid therapy $(n=14)$; ii) extensive excision $(n=21)$; and iii) local heat therapy groups $(n=39)$. Patients in the corticosteroid therapy group were administered $1 \mathrm{mg} / \mathrm{kg} / \mathrm{day}+$ glucocorticoid $+5 \mathrm{mg} /$ week methotrexate orally, and dosage reduction was started 2 weeks following the initiation of the treatment or the stabilization of the disease, with weekly reductions to the smallest dose that maintained stabilization of GLM. All patients received a course of glucocorticoids and methotrexate for $>3$ months. Patients in the extensive excision group underwent excision of the entire involved lobular system, including the posterior lacteal space with a gross margin clearance of $>1 \mathrm{~cm}$ of the normal glands. The tumor plastic technique (19) was used to repair any defects following the removal of the lesion if necessary. Patients in the local heat therapy group were requested to use the automated heating patch (20) on the lesion to maintain the temperature at $42-65^{\circ} \mathrm{C}$. For patients with multiple lesions, patches were applied for each lesion. Patches were not placed in direct contact with the skin to avoid burning and were changed every $3 \mathrm{~h}$ during the day and $6 \mathrm{~h}$ during the night. The treatment was maintained until the disappearance of local symptoms was confirmed via physical examination and ultrasound or MRI.

Remission was defined as the disappearance of local symptoms in the breast, including redness, swelling, pain and fistulas, and reduction in systemic symptoms, such as fever. In addition, no inflammatory lesions were to be observed by ultrasound examination.

Criteria for no response to treatment. The following criteria were used to determine no response to treatment: i) No response to either corticosteroid or local heat therapy was defined as a reduction in the lesion of $<20 \%$, an increase in the volume of measurable lesions of $>25 \%$ or the appearance of any new lesions following 3 months of continuous treatment; or ii) the appearance of any new lesions within the original lesion within 1 month after extensive excision.

Definition of recurrence. The following definition of recurrence was used in the present study for both the corticosteroid and local heat therapy groups: Emergence of new lesion(s) within the range of the primary location or any other part of the ipsilateral breast 1 month following the termination of therapy.

The following definition of recurrence was used in the present study for the extensive excision group: The detection of new lesion(s) within the range of the primary location or any other part of the ipsilateral breast by ultrasound 1 month following extensive excision.
Bacterial cultivation and identification. Tween80 was added to a common blood plate to make a high-fat blood plate. Pus and blood from the newly diagnosed patients were evenly precoated on the plate at room temperature for $1 \mathrm{~min}$ and tissue was cut in to $1 \mathrm{~mm}$ pieces and placed on the plate within $6 \mathrm{~h}$ of collection. Pus and tissue samples were allowed to grow at $37^{\circ} \mathrm{C}$ for $48 \mathrm{~h}$. Bacterial samples were collected and sent to Life Corporation (Thermo Fisher Scientific, Inc.) for PCR and the products was purified and extracted for DNA sequencing and sequence alignment. Routine bacterial identification including fungi, mycobacteria, anaerobes or aerobes of tissue, blood and pus collected from the 74 patients was performed in the Germ Lab in The Second Affiliated Hospital of Guangzhou Medical University (Guangzhou, China).

Growth of Corynebacterium kroppenstedtii. Corynebacterium kroppenstedtii separated from the tissue was incubated in Luria-Bertani liquid medium (Sigma-Aldrich; Merck KGaA) at 32,37 or $42^{\circ} \mathrm{C}$ respectively for $72 \mathrm{~h}$. To determine the growth of the bacterium, the optical density at $600 \mathrm{~nm}$ of the suspension was measured hourly using a spectrophotometer system (Tristar ${ }^{2}$ SLB942; Berthold Technologies) according to the manufacturer's protocol.

Statistical analysis. SPSS 21.0 software (IBM Corp.) was used to create the database and $\mathrm{R}$ software was used for statistical analysis. Clinical characteristics were descirbed using median (and Interquartile range, IQR) or number (and percent) as appropriate. $\chi^{2}$ tests were used to evaluate the differences in remission and recurrence rates across the three groups, and used the Kruskal-Wallis test to determine the intergroup differences in Corynebacterium kroppenstedtii growth, the remission time. Dunn's test was used following the Kruskal-Wallis for post-hoc pairwise comparisons, with P-values adjusted using the Benjamini-Hochberg method. Estimates with $\mathrm{P}<0.05$ were considered statistically significant.

\section{Results}

Patient characteristics. A total of 74 patients were included in the present study, all of whom were female; the median follow-up time was 29.5 months (total range, 8-123 months) and the median age was 31.5 years (range, 20-50 years), Among the patients, 66/74 (89.1\%) were married and had children, 5/74 (6.8\%) were married but childless and 3/74 (4.1\%) were unmarried and nulliparous. A total of 9/74 (13.2\%) had a history of oral contraceptive use and 16/74 (21.6\%) did not lactate normally for reasons including nipple inversion or reduced milk secretion (Table I).

Clinical presentations were well balanced among the treatment groups. The median maximum diameter of the lesion in the local heat group was higher compared with that in the corticosteroid and excision groups $(5.00 \mathrm{~cm}$ vs. $4.20 \mathrm{~cm}$ and $3.70 \mathrm{~cm}$, respectively); however, the differences between the groups were not significant. In addition, in $22(56.41 \%)$ patients in the local heat group, $8(57.14 \%)$ patients in the corticosteroid group and $9(42.86 \%)$ patients in the extensive excision group, multiple lesions were discovered. Furthermore, 7 (50.00\%) patients in the corticosteroid group, $16(76.19 \%)$ patients in the extensive excision group and $20(51.28 \%)$ patients in the local 
Table I. Baseline characteristics of the patients $(n=74)$.

\begin{tabular}{|c|c|}
\hline Parameter & Value \\
\hline \multicolumn{2}{|l|}{ Age (years) } \\
\hline Median (range) & $-31.5(20-50)$ \\
\hline$\leq 25$ & $8(10.8)$ \\
\hline $25-29$ & $20(27.0)$ \\
\hline $30-34$ & $26(35.1)$ \\
\hline $35-40$ & $13(17.6)$ \\
\hline$>40$ & $7(9.5)$ \\
\hline $\begin{array}{l}\text { Median follow-up time (range), } \\
\text { months }\end{array}$ & 29.5 (a total of 8-123) \\
\hline \multicolumn{2}{|l|}{ Marital or childbearing status } \\
\hline Married and had children & $66(89.1)$ \\
\hline Married but childless & $5(6.8)$ \\
\hline Unmarried and without children & $3(4.1)$ \\
\hline Unmarried with children & $0(0)$ \\
\hline History of oral contraceptives & $9(13.2)$ \\
\hline \multicolumn{2}{|l|}{ Lactation } \\
\hline Normal & $58(78.3)$ \\
\hline Abnormal in either breast & $16(21.6)$ \\
\hline Abnormal in the diseased breast & $9(12.2)$ \\
\hline \multicolumn{2}{|l|}{ Location of lesion } \\
\hline Left breast & $39(52.7)$ \\
\hline Right breast & $29(39.2)$ \\
\hline Bilateral breasts & $6(8.1)$ \\
\hline \multicolumn{2}{|l|}{ Involved quadrant } \\
\hline Upper external & $19(25.7)$ \\
\hline Inferior external & $9(12.2)$ \\
\hline Upper internal & $20(27.0)$ \\
\hline Inferior internal & $10(13.5)$ \\
\hline$>2$ & $16(21.6)$ \\
\hline \multicolumn{2}{|c|}{$\begin{array}{l}\text { Time interval between birth and onset } \\
\text { of GLM (years) }\end{array}$} \\
\hline$\leq 3$ & $50(67.6)$ \\
\hline$>3$ & $24(32.4)$ \\
\hline
\end{tabular}

Values are expressed as $\mathrm{n}(\%)$ unless otherwise indicated. GLM, granulomatous lobular mastitis.

heat group had no abscesses. However, there were no significant differences in the proportion of patients with multiple lesions and abscesses among the three groups (Table II).

Growth of Corynebacterium kroppenstedtii at different temperatures. Routine bacterial culture was performed to identify the pathogenic bacteria in the tissue, blood and pus of all patients of the present study, but no bacteria, including fungi, mycobacteria, anaerobes or aerobes, were detected in $64 / 74(86.5 \%)$ patients of the present study. The other patients were confirmed to have gram-positive bacterial infections (13.5\%). Corynebacterium kroppenstedtii was discovered to be the major pathogenic bacterium present in the tissue and pus of 11 patients with GLM (data not shown). In addition,

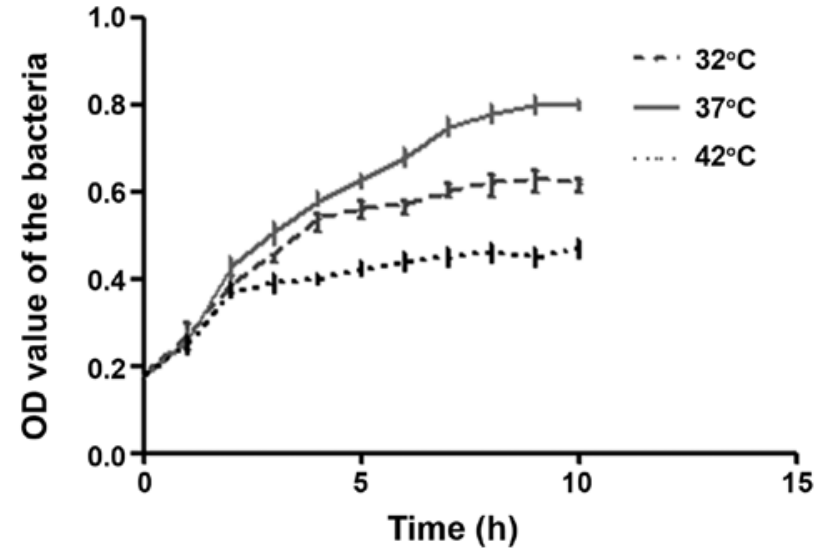

Figure 1. Corynebacterium kroppenstedtii suspension was incubated at 32 , 37 or $42^{\circ} \mathrm{C}$ for $10 \mathrm{~h}$. The number of Corynebacterium kroppenstedtii was markedly increased following incubation at $37^{\circ} \mathrm{C}$ for $10 \mathrm{~h}$; however, growth arrest was observed from $2 \mathrm{~h}$ of incubation at $42^{\circ} \mathrm{C}$. Significant differences in the OD value between different groups were present $(\mathrm{P}<0.05)$, and the difference was most noticeable between 37 and $42^{\circ} \mathrm{C}(\mathrm{P}=0.020)$. OD, optical density.

it was previously suggested that Corynebacterium kroppenstedtii may be responsible for multiple recurrence of GLM, and that the inhibition of Corynebacterium kroppenstedtii may help to control GLM (17). Therefore, the present study determined the optimum temperature at which the growth of the bacteria was inhibited in vitro. the results revealed that the optimum growth temperature of Corynebacterium kroppenstedtii was $37^{\circ} \mathrm{C}$, while the growth at $42^{\circ} \mathrm{C}$ was markedly inhibited (Fig. 1).

Comparison of first remission rates among the different treatments. The first remission rate was compared between the three groups; among the 14 patients treated with corticosteroids, 12 reached first remission and 2 were non-responsive to the treatment $(85.7 \%)$. Among the 39 patients treated with local heat therapy, 30 cases were cured, while 9 cases were not cured (76.9\%). Among the 21 patients who received expanded excision, 19 reached remission and 2 did not, and consequently, the remission rate was $90.4 \%$. There were no significant differences in the remission rates among the three groups $(\mathrm{P}=0.221$; Table III).

Comparison of recurrence rates among the three different treatments. As for the recurrence rate, the corticosteroid group had one case of recurrence $(8.33 \%)$, the expanded excision group had two cases of recurrence (10\%) and three cases of recurrence $(10 \%)$ were observed in the local heat therapy group. However, no significant differences in the recurrence rates were obtained among the three groups $(\mathrm{P}=0.985$; Table IV).

Comparison of first remission time between corticosteroid therapy and local heat therapy. The median remission time (treatment duration: Time from initiation of treatment to the first-time remission) between the corticosteroid and local heat therapy groups was investigated. The patients receiving local heat therapy had a significantly reduced remission time compared with those receiving corticosteroids (5.30 months vs. 11.27 months; $\mathrm{P}=0.016$; Fig. 2). 
Table II. Clinical characteristics of the patients in the three groups.

\begin{tabular}{lcccr}
\hline Parameter & Corticosteroids $(\mathrm{n}=14)$ & Extensive excision $(\mathrm{n}=21)$ & Local heat therapy $(\mathrm{n}=39)$ & P-value \\
\hline Age (years) & $30.93 \pm 5.64$ & $32.86 \pm 6.23$ & $32.10 \pm 5.90$ & 0.645 \\
${\text { Maximum diameter }(\mathrm{cm}){ }^{\mathrm{a}}}^{\text {Multiplicity of lesions }}{ }^{\mathrm{b}}$ & $4.56 \pm 1.95$ & $3.97 \pm 2.37$ & $5.11 \pm 2.01$ & 0.141 \\
$\quad$ Multiple & $8(57.14)$ & $9(42.86)$ & & 0.590 \\
Single & $6(42.86)$ & $12(57.14)$ & $17(56.41)$ & \\
Abscess & & & & \\
No & $7(50.00)$ & $16(76.19)$ & $20(51.28)$ & \\
Yes & $7(50.00)$ & $5(23.81)$ & $19(48.72)$ & \\
\hline
\end{tabular}

Age and lesion-associated information is provided in Table II. ${ }^{\mathrm{a} M e a s u r e d ~ b y ~ u l t r a s o u n d ~ o r ~ M R I . ~}{ }^{\mathrm{b}}$ Patients with single or multiple lesions was examined by ultrasound or MRI. 'Ultrasound or MRI was performed to determine the presence of abscess in the lesion. Values are expressed as the mean \pm standard deviation or $\mathrm{n}(\%)$. The average age and lesion diameter were compared across the three groups using one-way ANOVA, while the percentage of multi-lesion and abscess cases was compared using a $\chi^{2}$-test.

Table III. Comparison of remission rates among three different treatments.

\begin{tabular}{lcccc}
\hline Treatment & Reached first remission (n) & No. remission (n) & Total (n) & Remission rate (\%) \\
\hline Corticosteroids & 12 & 2 & 14 & 85.7 \\
Extensive excision & 19 & 2 & 21 & 90.4 \\
Local heat therapy & 30 & 9 & 39 & 76.9 \\
\hline
\end{tabular}

The $\chi^{2}$-test was used to analyze the differences in remission rates among the three groups but no significant difference was obtained ( $\left.\mathrm{P}=0.221\right)$.

Table IV. Comparison of recurrence among three different treatments.

\begin{tabular}{lccc}
\hline Treatment & Recurrence $(\mathrm{n})$ & Primary healing $(\mathrm{n})$ & Recurrence rate $(\%)$ \\
\hline Corticosteroids & 1 & 12 & 8.33 \\
Extensive excision & 2 & 19 & 10.0 \\
Local heat therapy & 3 & 30 & 10.0 \\
\hline
\end{tabular}

The $\chi^{2}$-test was used to analyze the differences in recurrence rates among the three groups, no significant difference $(\mathrm{P}=1.000)$.

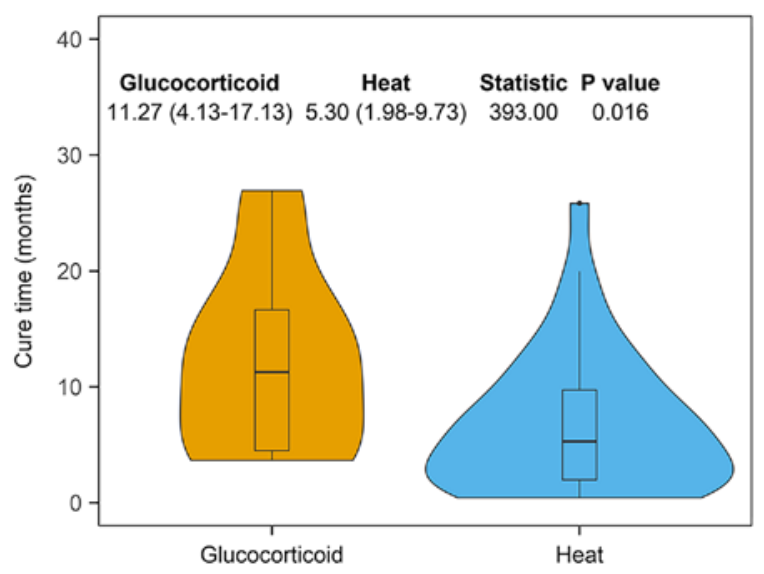

Figure 2. Remission time in the corticosteroid and local heat therapy groups. The median remission time was 11.27 and 5.30 months, respectively. According to a Mann-Whitney U-test, there was a significant difference in the remission time between the two groups $(\mathrm{P}<0.05)$.
Side effects. The major side effects observed in the patients with local heat therapy were redness of the skin and a mild rash (Table SI), which did not require extra treatment.

\section{Discussion}

GLM is a benign disorder of the breast that is frequent in females of child-bearing age, but not during the lactation period (21). Regarding its occurrence, there is no preferential side of the breast and it is usually unilateral instead of bilateral (22). The etiology of GLM has yet to be fully elucidated; however, previous studies have suggested that it may be associated with the following factors: i) Autoimmunity. Kessler and Wolloch (23) first hypothesized that GLM was a type of autoimmune disease, which was later proved by the fact that the size of the lumps may be reduced by treatment with glucocorticoids (24); ii) Infection, trauma, physical or chemical factors. 
Fletcher et al (25) proposed that trauma, local breast infection and other physical and chemical factors may stimulate the breast duct and gland cavity to secrete milk or cause the exfoliation of keratinized epithelium to the lobule, both of which may lead to an inflammatory reaction in the lobules of the mammary gland; and iii) Corynebacterium infection. In 2002, Paviour et al (26) discovered that Corynebacterium kroppenstedtii may be cultured from the non-lactating mastitis tissue of 13/24 patients. In addition, in 2003, Taylor et al (27) reported a Corynebacterium kroppenstedtii infection in 27/34 patients with GLM. In further studies, Corynebacterium kroppenstedtii was also confirmed; Wong et al (28) used matrix-assisted laser desorption/ionization-time-of-flight mass spectrometry to confirm that Corynebacterium kroppenstedtii was the only pathogenic bacteria in 39/42 (92.9\%) cases. A bioinformatics analysis by $\mathrm{Yu}$ et al (15) revealed that Corynebacterium was present in patients with GLM, with gene identification of Corynebacterium kroppenstedtib ranging from 1.1 to $58.9 \%$ and the predominance of Corynebacterium kroppenstedtii infection $(11 / 19,57.9 \%)$. The predominance of Corynebacterium kroppenstedtiib-associated breast specimens in the present study was consistent with that reported in previous studies $(15,28)$. Other factors reported included the use of oral contraceptives $(29,30)$, breast-feeding related events $(31,32)$, smoking (33) and $\alpha 1$-antitrypsin deficiency (34).

As the in vitro experiment of the present study indicated that the optimum growth temperature for Corynebacterium kroppenstedtii was $37^{\circ} \mathrm{C}$ and its growth was notably inhibited at $42^{\circ} \mathrm{C}$, local temperature control may result in growth arrest of Corynebacterium kroppenstedtii. Local heat therapy has been evaluated as a treatment for inflammation and the acceleration of wound healing; however, to the best of our knowledge, the present study was the first trial to assess local heat therapy as a treatment for GLM. Of note, 30 patients achieved first remission (76.9\%), with no significant differences compared with the corticosteroid therapy and expanded excision groups. Concurrently, the healing time following local heat therapy was only 5.9 months, which was a shorter time compared with that following corticosteroid therapy. Despite taking into consideration the gradual tapering time of corticosteroids during treatment, the differences in the median healing time between the two groups remained $>6$ months, suggesting that the differences in the healing time between local heat therapy and corticosteroids may not only be explained by the long course of steroids withdrawal, but by the advantages of local heat therapy itself. Of note, in the present study, the presence of Corynebacterium was confirmed in $11 / 15$ patients diagnosed with GLM in both the affected tissue and pus.

It has been suggested that patients with GLM should not opt for surgery, as the recurrence rate may be as high as $50 \%(35,36)$. In addition, the inevitable complications, such as breast deformation and poor wound healing, limit the application of surgery (9). Regarding the cause of recurrence, previous studies have ascribed the incompleteness of excision to the failure of surgical treatment and put forward the concept of extensive excision of the lesion $(8,37)$, which has been suggested by several studies as a critical treatment for GLM, demonstrating an instant remission and providing a comprehensive pathological diagnosis $(8,38)$. Schelfout et al $(34)$ reported a success rate of $90.3 \%$ and a recurrence rate of
$8.7 \%$ with surgical intervention. In this previous study, extensive excision was defined as the excision of the lesion and the surrounding normal gland tissue; the patients experienced little recurrence, as the lesions reported were mainly a single mass and relatively small, with an average size of $3.8 \pm 2.3 \mathrm{~cm}$. In the present study, a cure rate of $90.4 \%$ was achieved with expanded excision. The possible reasons for this cure rate were that a total of $10(47.6 \%)$ cases in this group presented with single, relatively small lumps $(<3 \mathrm{~cm})$, which were easy to surgically remove, and that one patient opted for mastectomy and breast reconstruction; furthermore, the cure mentioned here refers to the first remission, which was defined as the disappearance of local symptoms by physical examination and imaging examination lasting for 1 month after the operation. The application of oncoplastic techniques also makes extensive resection feasible which may lead to a low recurrence rate. Therefore, these results suggested that surgery may be suitable for patients with GLM with relatively small single lumps. Surgery may also be considered for larger lesions, if the surgeon is skilled in the application of oncoplastic techniques.

A high recurrence rate is one of the features of GLM that make it challenging to manage, and recurrence has been reported for all currently available therapeutic options, including surgical resection and oral steroids (39). In the present study, certain patients in the corticosteroid, extensive excision and local heat therapy groups all experienced recurrence, with rates of $8.3,10.0$ and $10.0 \%$, respectively. However, no significant differences were present among the three groups. Thus, the recurrence rate in patients receiving local heat therapy was not increased compared with that in patients who had received extensive excision or corticosteroid therapy, which suggested that local heat therapy may not be inferior to the other two approaches in terms of recurrence.

The major side effects of local heat therapy were redness of the skin, a mild rash and the requirement to change the hot patch frequently. However, all patients were able to tolerate the above side effects; none of the 39 patients who received local heat therapy developed severe rash or severe burns to the skin on the breast. In the corticosteroid group, concentric obesity, abnormal glucose tolerance and facial acne were the most common side effects. Expanded excision is not widely used at the early stage of GLM due to the poor postoperative appearance of the breast; however, the application of oncoplastic surgery has made surgical excision a more popular option for patients with GLM (40). However, due to the loss of a large volume of the affected breast, contralateral breast reduction surgery is at times indicated, providing a dilemma for patients and clinicians. By contrast, local heat therapy does not markedly affect the appearance and volume of the breast, and even if the treatment fails, it does not cause any severe psychological trauma in the patients. However, in certain patients, the remaining local malformation due to ulceration in the local heat therapy group may still require surgical adjustment.

As a limitation of the present study, the combination of local heat therapy with surgery or corticosteroids therapy was not assessed. It is clear from the present study that extensive excision caused huge tissue losses in the patients and recurrence were more frequently observed in the local heat therapy group than in the other two groups, As such, it is possible that extensive excision following local heat therapy could reduce 
the resection range. While corticosteroid therapy may achieve a prompt response to reduce the local symptoms, whether the combination of local heat therapy and corticosteroids could provide rapid control of local manifestations and reach a quick remission requires to be further investigated.

Due to the lack of response to routine anti-inflammatory therapies, high recurrence rates and adverse effects of the current treatments, GLM remains a refractory disease. To the best of our knowledge, the present study was the first to investigate local heat therapy as a treatment option for patients with GLM, which demonstrated certain advantages over extensive excision and corticosteroid therapy. Thus, compared with standard treatments, local heat therapy may be a promising option to explore due to its low cost, relatively fewer side effects and similar cure rate, as well as feasibility for all patients.

\section{Acknowledgements}

Not applicable.

\section{Funding}

The present was supported by grants from the Natural Science Foundation of Guangdong Province (grant no. 2018A030310184). This work was also supported by the Youth Program of the National Natural Science Foundation of China (grant no. 81802817).

\section{Availability of data and materials}

The datasets used and/or analyzed during the current study are available from the corresponding author on reasonable request.

\section{Authors' contributions}

LZ contributed to study design and coordination, and supervision of the study. XC performed analysis and interpretation of data; generated all figures and tables; conducted most of the experiments; drafted and revised the manuscript. QY participated in data collection and patient follow-up. WZ was responsible for all statistical analyses. XH, TX TC and HJ performed sample collection. All authors read and approved the final manuscript.

\section{Ethics approval and consent to participate}

This study was approved by the Ethics Review Board of Guangzhou Medical University and the Ethics Review Board of The Second Affiliated Hospital of Guangzhou Medical University (Guangzhou, China). Written informed consent was provided by the study participants and/or their legal guardians.

\section{Patient consent for publication}

Not applicable

\section{Competing interests}

The authors declare that they have no competing interests.

\section{References}

1. Hwang MJ, Rogers A and Vidya R: Idiopathic granulomatous mastitis: Rare but important. BMJ Case Rep 2010: bcr1020092334, 2010 .

2. Altintoprak F, Kivilcim T and Ozkan OV: Aetiology of idiopathic granulomatous mastitis. World J Clin Cases 2: 852-858, 2014.

3. Aslan H, Pourbagher A and Colakoglu T: Idiopathic granulomatous mastitis: Magnetic resonance imaging findings with diffusion MRI. Acta Radiol 57: 796-801, 2016.

4. Mahmodlou R, Dadkhah N, Abbasi F, Nasiri J and Valizadeh R: Idiopathic granulomatous mastitis: Dilemmas in diagnosis and treatment. Electron Physician 9: 5375-5379, 2017.

5. Benson JR and Dumitru D: Idiopathic granulomatous mastitis: Presentation, investigation and management. Future Oncol 12: 1381-1394, 2016.

6. Yalcin KehribarD, Izci Duran T and Ozgen M: AB1052 Evaluation of symptoms, depression and anxiety levels in young women with idiopathic granulomatous mastitis. Ann Rheum Dis 79: 1816-1816, 2020.

7. Ahmed YS and Abd El Maksoud W: Evaluation of therapeutic mammoplasty techniques in the surgical management of female patients with idiopathic granulomatous mastitis with mild to moderate inflammatory symptoms in terms of recurrence and patients' satisfaction. Breast Dis 36: 37-45, 2016.

8. Yabanoglu H, Colakoglu T, Belli S, Aytac HO, Bolat FA, Pourbagher A, Tezcaner T, Yildirim S and Haberal M: A comparative study of conservative versus surgical treatment protocols for 77 patients with idiopathic granulomatous mastitis. Breast J 21: 363-369, 2015.

9. Mizrakli T, Velidedeoglu M, Yemisen M, Mete B, Kilic F, Yilmaz H, Ozturk T, Ozaras R, Aydogan F and Perek A: Corticosteroid treatment in the management of idiopathic granulomatous mastitis to avoid unnecessary surgery. Surg Today 45: 457-465, 2015.

10. Pandey TS, Mackinnon JC, Bressler L, Millar A, Marcus EE and Ganschow PS: Idiopathic granulomatous mastitis-a prospective study of 49 women and treatment outcomes with steroid therapy. Breast J 20: 258-266, 2014.

11. Altintoprak F, Kivilcim T, Yalkin O, Uzunoglu Y, Kahyaoglu Z and Dilek ON: Topical steroids are effective in the treatment of idiopathic granulomatous mastitis. World J Surg 39: 2718-2723, 2015.

12. Farouk O, Abdelkhalek M, Abdallah A, Shata A, Senbel A, Attia E, Elghaffar MA, Mesbah M, Soliman N, Amin M and El-Tantawy D: Rifampicin for idiopathic granulomatous lobular mastitis: A promising alternative for treatment. World J Surg 41: 1313-1321, 2017.

13. Al-Jarrah A, Taranikanti V, Lakhtakia R, Al-Jabri A and Sawhney S: Idiopathic granulomatous mastitis: Diagnostic strategy and therapeutic implications in Omani patients. Sultan Qaboos Univ Med J 13: 241-247, 2013.

14. Kaviani A, Vasigh M, Omranipour R, Mahmoudzadeh H, Elahi A, Farivar L and Zand S: Idiopathic granulomatous mastitis: Looking for the most effective therapy with the least side effects according to the severity of the disease in 374 patients in Iran. Breast J 25: 672-677, 2019.

15. Yu HJ, Deng H, Ma J, Huang SJ, Yang JM, Huang YF, Mu XP, Zhang $\mathrm{L}$ and Wang Q: Clinical metagenomic analysis of bacterial communities in breast abscesses of granulomatous mastitis. Int J Infect Dis 53: 30-33, 2016.

16. Tauch A, Fernandez-Natal I and Soriano F: A microbiological and clinical review on Corynebacterium kroppenstedtii. Int J Infect Dis 48: 33-39, 2016.

17. Johnson MG, Leal S, Plongla R, Leone PA and Gilligan PH: The brief case: Recurrent granulomatous mastitis due to Corynebacterium kroppenstedtii. J Clin Microbiol 54: 1938-1941, 2016.

18. Schmidt KL, Ott VR, Rocher G and Schaller H: Heat, cold and inflammation. Z Rheumatol 38: 391-404, 1979.

19. Mathapati SN, Goel A, Mehta S, Aggarwal J, Aravindan R, Nayak V, Panda SK, Pande PK and Kumar K: Oncoplastic breast reconstruction in breast conservation surgery: Improving the oncological and aesthetic outcomes. Indian J Surg Oncol 10: 303-308, 2019.

20. Klarzak I, Ura-Bińczyk E, Płocińska M and Jurczyk-Kowalska M: Effect of temperature and humidity on heat effect of commercial chemical warmers based on iron powder. Therm Sci Eng Prog 6: 87-94, 2018. 
21. Mathew M, Siwawa P and Misra S: Idiopathic granulomatous mastitis: An inflammatory breast condition with review of the literature. BMJ Case Rep 2015: bcr2014208086, 2015.

22. Atak T, Sagiroglu J, Eren T, Ali Ozemir I and Alimoglu O: Strategies to treat idiopathic granulomatous mastitis: Retrospective analysis of 40 patients. Breast Dis 35: 19-24, 2015.

23. Kessler E and Wolloch Y: Granulomatous mastitis: A lesion clinically simulating carcinoma. Am J Clin Pathol 58: 642-646, 1972.

24. Allen SG, Soliman AS, Toy K, Omar OS, Youssef T, Karkouri M, Ayad E, Abdel-Aziz A, Hablas A, Tahri A, et al: Chronic mastitis in egypt and morocco: Differentiating between idiopathic granulomatous mastitis and IgG4-related disease. Breast J 22: 501-509, 2016.

25. Fletcher A, Magrath IM, Riddell RH and Talbot IC: Granulomatous mastitis: A report of seven cases. J Clin Pathol 35: 941-945, 1982.

26. Paviour S, Musaad S, Roberts S, Taylor G, Taylor S, Shore K, Lang S and Holland D: Corynebacterium species isolated from patients with mastitis. Clin Infect Dis 35: 1434-1440, 2002.

27. Taylor GB, Paviour SD, Musaad S, Jones WO and Holland DJ: A clinicopathological review of 34 cases of inflammatory breast disease showing an association between corynebacteria infection and granulomatous mastitis. Pathology 35: 109-119, 2003.

28. Wong SCY,Poon RWS, Chen JHK, Tse H,Lo JYC,Ng TK, Au JCK, Tse CWS, Cheung IYY, Yuk MT, et al: Corynebacterium kroppenstedtii is an emerging cause of mastitis especially in patients with psychiatric illness on antipsychotic medication. Open Forum Infect Dis 4: ofx096, 2017.

29. Oran ES, Gurdal SO, Yankol Y, Öznur M, Calay Z, Tunacı M and Soybir GR: Management of idiopathic granulomatous mastitis diagnosed by core biopsy: A retrospective multicenter study. Breast J 19: 411-418, 2013.

30. Gurleyik G, Aktekin A, Aker F, Karagulle H and Saglamc A Medical and surgical treatment of idiopathic granulomatous lobular mastitis: A benign inflammatory disease mimicking invasive carcinoma. J Breast Cancer 15: 119-123, 2012.
31. Al-Khaffaf B, Knox F and Bundred NJ: Idiopathic granulomatous mastitis: A 25-year experience. J Am Coll Surg 206: 269-273, 2008

32. Sakurai K, Fujisaki S, Enomoto K, Amano S and Sugitani M Evaluation of follow-up strategies for corticosteroid therapy of idiopathic granulomatous mastitis. Surg Today 41: 333-337, 2011.

33. Ozel L, Unal A, Unal E, Kara M, Erdoğdu E, Krand O, Günes P, Karagül H, Demiral S and Titiz MI: Granulomatous mastitis: Is it an autoimmune disease? Diagnostic and therapeutic dilemmas. Surg Today 42: 729-733, 2012.

34. Schelfout K, Tjalma WA, Cooremans ID, Coeman DC, Colpaert CG and Buytaert PM: Observations of an idiopathic granulomatous mastitis. Eur J Obstet Gynecol Reprod Biol 97: 260-262, 2001

35. Hovanessian Larsen LJ, Peyvandi B, Klipfel N, Grant E and Iyengar G: Granulomatous lobular mastitis: Imaging, diagnosis, and treatment. AJR Am J Roentgenol 193: 574-581, 2009.

36. Lin CH, Hsu CW, Tsao TY and Chou J: Idiopathic granulomatous mastitis associated with risperidone-induced hyperprolactinemia. Diagn Pathol 7: 2, 2012.

37. Zhang Y, Zhou Y, Mao F, Guan J and Sun Q: Clinical characteristics, classification and surgical treatment of periductal mastitis. J Thorac Dis 10: 2420-2427, 2018.

38. Li J: Diagnosis and treatment of 75 patients with idiopathic lobular granulomatous mastitis. J Invest Surg 32: 414-420, 2019.

39. Lei X, Chen K, Zhu L, Song E, Su F and Li S: Treatments for idiopathic granulomatous mastitis: Systematic review and meta-analysis. Breastfeed Med 12: 415-421, 2017.

40. Hladik M, Schoeller T, Ensat F and Wechselberger G: Idiopathic granulomatous mastitis: Successful treatment by mastectomy and immediate breast reconstruction. J Plast Reconstr Aesthet Surg 64: 1604-1607, 2011. 